\title{
Load Reduction in Wind Turbines with Smart Rotors Using Trial Varying Iterative Learning Control Law
}

\author{
Weronika N. Nowicka*, Bing Chu*, Owen R. Tutty**, and Eric Rogers*
}

\begin{abstract}
Control of aerodynamic loads is a crucial issue in keeping wind energy economically competitive with traditional energy sources. This paper continues the investigation of the use of Iterative Learning Control (ILC) for load control in wind turbines with smart devices on rotor blades. A relatively simple computational fluid dynamics model is used to simulate the flow past an airfoil. Assuming no actuator delay, fluctuations in load due to periodic disturbances can be almost completely eliminated by a simple ILC control scheme. In application, however, delay due to the actuators will arise and could have a negative effect on real-time performance and influence the desired attenuation. This paper develops a novel method of using trial varying ILC to improve aerodynamic load performance in the presence of actuator dynamics. The results of a simulation based assessment of the new control system are also given and areas for possible future research discussed.
\end{abstract}

\section{NOMENCLATURE}

$\begin{array}{ll}i & \text { Trial number } \\ j, k, k_{c} & \text { Step indices } \\ u & \text { Control input signal } \\ e, E & \text { Error signal } \\ \mu & \text { Controller gain } \\ z, \zeta & \text { Complex variables } \\ t, \Delta t & \text { Time and time step } \\ H & \text { Chord length } \\ V_{\infty} & \text { Mean free stream velocity } \\ \mathbf{x}=(x, y) & \text { Coordinates } \\ \mathbf{v}=\left(v_{x}, v_{y}\right) & \text { Velocity components } \\ \Gamma & \text { Circulation } \\ A & \text { Amplitude of oscillations } \\ T & \text { Period of oscillations } \\ L & \text { Lift } \\ \delta & \text { Controller's phase shift } \\ \lambda & \text { Speed of response coefficient } \\ \mathcal{L}_{2} & \text { 2-norm } \\ \mathcal{L}_{\infty} & \text { Infinity norm }\end{array}$

\section{INTRODUCTION}

Wind energy is a fast-growing field recognized as a cost effective and environmentally friendly energy source. This trend will continue as the limited fossil fuel reserve and energy security are one of the main public concerns nowadays.

*W. N. Nowicka, B. Chu, and E. Rogers are with Electronics and Computer Science, University of Southampton, Southampton SO17 1BJ, U.K. (e-mail: wnnlg12@soton.ac.uk; B.Chulsoton.ac.uk; etardsoton.ac.uk).

$* * O$. Tutty is with the Faculty of Engineering and the Environment, University of Southampton, Southampton SO17 1BJ, U.K. (e-mail ortasoton.ac.uk).
Over past three decades the typical size of wind turbine rotor has increased from $15 \mathrm{~m}$ to over $124 \mathrm{~m}$ [1]. Accordingly, the loads on the blades have increased. Passive control of such structures became ineffective and for that reason there exists a need of developing advanced control systems and strategies in order to control the aerodynamic loads. Aerodynamic load control of wind turbines involves altering the lift force on the rotor blades. This aim can be obtained in numerous ways: by varying flow velocity, blade incidence angle or blade length. The modern control approach includes modification of blade section aerodynamics and is called active flow control (AFC). Advanced active control devices such as trailing-edge flaps, microtabs, plasma actuators and vortex generators are called 'smart devices' or 'smart rotor control' [2].

\section{A. Wind Energy Costs}

A developing trend in wind energy provision is operate offshore reduction and hence cost is a crucial issue. In order to remain economically competitive with traditional energy sources the cost of energy (COE) should be lowered. Such cost is calculated using (1) [3], which is the ratio of the capital cost and operations and the maintenance cost (O\&M) to the total energy capture in a turbine's lifetime.

$$
C O E=\frac{\text { Capital Cost }+O \& M \text { costs }}{\text { Lifetime Energy Capture }}
$$

Possible ways of decreasing COE include increasing the turbine size and therefore the energy capture, reducing the amount of materials to lower the capital cost or reducing the downtime and O\&M costs by constructing more reliable turbines. Reduction of O\&M can be achieved by designing effective control systems for rotor loads that will provide a decrease in fatigue and extreme loads on the components. This, in turn, will reduce maintenance, improve system reliability and produce an increase in component lifespan.

\section{B. Smart Rotors}

One way to reduce the fatigue and extreme loads is to employ active flow control devices. Such devices are placed along the span of the rotor blade (e.g. on the trailingedge) (Fig. 1) and act by modifying the local flow and therefore the lift [4]. Fifteen AFC devices were detailed in [2] including traditional and nontraditional trailing-edge flaps, microtabs, vortex generators, and plasma actuators. Most of these devices have been implemented in other industry fields such as aircraft design or unmanned aerial vehicles (UAV). Some of them, e.g., trailing edge flaps and microtabs have shown the potential for wind turbine control in numerical 


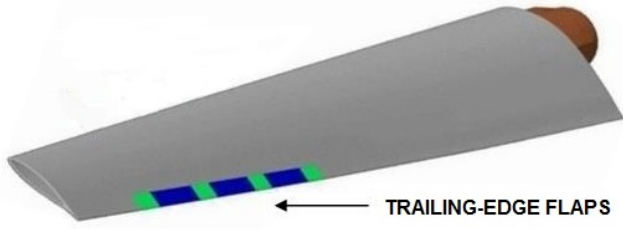

Fig. 1. AFC devices [4]

experiments and wind tunnel tests [3] [5] but still require further research and validation on full-scale turbines. Others, such as circulation control, have been tested for aircraft or UAVs [6] but are yet to be investigated for wind turbines. The main purpose of AFC devices is to alleviate excessive, highfrequency loads caused by turbulent winds and these devices can be used for sectional lift adjustment, drag reduction or noise suppression.

In a sophisticated wind turbine control system there will be a master controller responsible for pitching the blades and the individual controllers responsible for active flow control. Various approaches for control of smart rotors have been proposed ranging from Proportional plus Integral plus Derivative (PID) controllers to advanced techniques such as optimal control. A brief survey of the control design methods that could be possibly be used in this field is given in [8].

\section{Iterative Learning Control}

Iterative Learning Control (ILC) is a feedforward control approach used for systems operating in a repetitive manner, i.e., for systems that perform the same finite-time task over and over again. Each execution is commonly termed a trial (or pass) and the finite duration is known as the trial length. Once each trial is complete resetting to the starting location occurs ready for the start of the next trial. Once a trial is complete all information generated is available for use in constructing the control signal for the next trial and thereby improve performance from trial-to-trial. In fact, this strategy can also be applied to systems where there is a stoppage between successive executions of a task, i.e., it is not required to reset to a starting location.

The simplest form of ILC controller has the structure

$$
u_{i+1}(k)=u_{i}(k)+\mu e_{i}(k+1)
$$

where: $i-$ denotes the trial number; $u_{i}(k)$ - input signal at time step $k$ on trial $i ; \mu-$ the controller gain; $e_{i}(k)-$ the error at time step $k$ on trial $i$. The trial error on any trial is the difference between a pre-specified reference signal and the output on this trial. The ILC design problem can then be formulated in terms of ensuring convergence in $i$ of the sequence whose entries are the errors on the trials. One starting point for background on ILC, including controllers with dynamics, is the survey papers [9] and [10]. Successful applications of ILC include gantry robots, e.g., [11] and robotic-assisted upper limb stroke rehabilitation, e.g. [21].

\section{Rejection of Periodic Load Disturbances}

The load disturbances caused by effects such as wind shear, tower shadow or yaw misalignment depend on the speed and rotation angle of the blade [12]. The flow past the blade will therefore contain a periodic component which will become even larger when the rotor size increases. For that reason, ILC algorithm, which uses repetitive nature of processes, can be considered as potentially beneficial. The examination of ILC for improved aerodynamic load performance of wind turbines was a subject of the recent research [13] [14] [15]. The control system showed a good potential for the case with no (or a very small) actuation delay but in case of bigger delays the degradation of performance was observed.

This paper proposes the novel approach of using trial varying ILC to improve the aerodynamic load performance which is degraded due to actuator dynamics. The modelling of the flow and the airfoil generation process are presented in the next section. Subsequently, the control system and the simulation results are presented. The last section consist of conclusions, the critical review of this work and describes areas for future research.

\section{FLOW PAST AN AIRFOIL}

In this paper a relatively simple computational fluid dynamics (CFD) model is used to simulate the flow past an airfoil. The airfoil is generated using a conformal mapping.

\section{A. Airfoil profile}

Wide range of airfoils can be generated by applying Joukowski transform to a circle as in [17]. Varying the coordinates of the centre of the circle results in different airfoil shapes. However, airfoils obtained using Joukowsky transform have a cusp at the trailing edge. Kàrmàn-Trefftz transform is strictly connected to Joukowski transform and enables to generate airfoils with non-zero trailing edge angle $\alpha$ [17]. The profile of the airfoil can be calculated using

$$
z=n \frac{\left(1+\frac{1}{\zeta}\right)^{n}+\left(1-\frac{1}{\zeta}\right)^{n}}{\left(1+\frac{1}{\zeta}\right)^{n}-\left(1-\frac{1}{\zeta}\right)^{n}}
$$

where $z=x+i y$ is a complex variable in the new space (airfoil profile) and $\zeta=\chi+i \eta$ is a variable in original space (circle). Parameter $n$ is slightly smaller than 2 and the trailing edge angle $\alpha$ is related to $n$ by $n=2-\alpha / \pi$. In this work the parameter $n=1.9$ and the coordinates of the centre of the circle $x_{0}=-0.05$ and $y_{0}=0.2$ are used to generate the profile. The resulting airfoil is given in Fig. 2. The airfoil is normalized using the chord length.

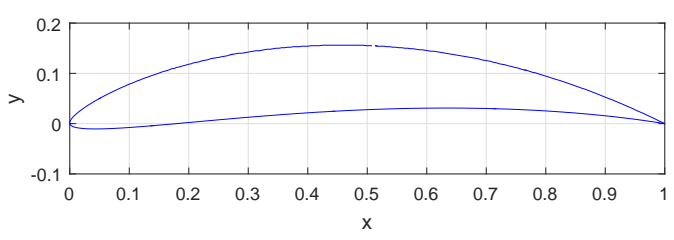

Fig. 2. Airfoil profile 


\section{B. Flow model}

The same flow model as in the previous work [15] is used in this research. The model includes the wake generated from the trailing edge (vorticity is shed into the flow from the trailing edge) and assumes inviscid dynamics of the flow. Such a representation is adequate for preliminary investigation of the control algorithms that could be used, when fully developed, in future experiments and is less expensive computationally than full Navier-Stokes simulation.

As the velocity and chord length vary along the blade the time $t$ for the flow to pass the blade section changes as well. In this paper this time is considered as non-dimensional using $t^{*}=\frac{H}{V_{\infty}} t$, where $H$ denotes the chord length, $V_{\infty}$ denotes the mean free stream velocity, $t^{*}$ is dimensional and $t$ is non-dimensional.

In order to satisfy the boundary conditions a panel method [16] with $N$ vortex and $N$ source panels is used. In this work 400 vortex panels clustered towards the trailing edge are placed just above the surface and 400 source panels are placed just below the surface. The system of equations consists of $2 N$ equations and $2 N$ unknowns corresponding to each panel. The additional condition resulting from Kelvins circulation theorem is used to make the system over-determined so the solution can be obtained using leastsquare approximation. This theorem states that the circulation around a closed curve moving with the fluid remains constant with time.

Finally, the flow consists of the free-stream component $\mathbf{V}_{0}$, velocity field which is generated by $N$ vortex panels $\mathbf{v}_{p}(\mathbf{x}, t)=\left(v_{p x}, v_{p y}\right)$ and discrete vortices that are shed into the wake. Moreover, disturbances are introduced into the flow in the form of $M$ discrete vortices as described in [19]. The governing equation for a $2 \mathrm{D}$ inviscid incompressible fluid is

$$
\frac{D \omega}{D t}=\frac{\partial \omega}{\partial t}+v_{x} \frac{\partial \omega}{\partial x}+v_{y} \frac{\partial \omega}{\partial y}=0
$$

where $D / D t=\partial / \partial t+v_{x} \partial / \partial x+v_{y} \partial / \partial y$ denotes the material derivative and $\omega=\partial v_{y} / \partial x-\partial v_{x} / \partial y$ denotes the vorticity. This equation states that a collection of discrete vortices is convected with the flow and every individual vortex moves according to

$$
\frac{d \mathbf{x}_{v}}{d t}=\mathbf{v}\left(\mathbf{x}_{v}, t\right)
$$

where $\mathbf{x}_{v}$ denotes the position and the velocity field of an individual vortex $\mathbf{v}_{v j}$ is given by

$$
\mathbf{v}_{v j}=\Gamma_{j} \frac{\left(-\left(y-y_{v j}\right), x-x_{v j}\right)}{\left|\mathbf{x}-\mathbf{x}_{v j}\right|^{2}} F\left(\left|\mathbf{x}-\mathbf{x}_{v j}\right|\right)
$$

where $\Gamma_{j}$ denotes the strength of the vortex and $F(s)=$ $\int_{0}^{s} \gamma(s) d s$, where $\gamma(s)$ is the Gaussian distribution function. Finally, the complete velocity field is given by

$$
\mathbf{v}(\mathbf{x}, t)=\mathbf{V}_{0}+\mathbf{v}_{p}+\mathbf{v}_{v}
$$

where $\mathbf{v}_{v}=\sum_{j=1}^{M} \mathbf{v}_{v j}$.

Summarizing at this stage, a non-linear system of equations is produced due to the interaction between the vortices and the body. A numerical solution to the problem can be obtained using second-order Runge-Kutta method [19]. This model was validated in previous work [15] by comparing it with the standard source [20].

The smart devices are modelled by modifying the circulation on the trailing edge and thereby the lift. At every time step a new vortex with strength

$$
\Gamma_{n}=-u
$$

is generated, where $u$ denotes the control input signal and the lift calculated from the pressure distribution on the body surface is the output. Altering the circulation on the trailing edge represents devices such as flaps or microtabs which also act by generating vortices or changing the flow on the trailing edge. The next section applies this model to ILC for load management on wind turbines by smart rotors.

\section{ILC BASED LOAD REDUCTION}

The model presented in Section II is used to examine ILC schemes for load control of wind turbines with smart rotors.

\section{A. Load Control in Wind Turbines with Smart Rotors}

Assuming no vortices the flow past an airfoil is periodic with velocity equal to

$$
V_{0 x}=1+A \sin \left(\frac{2 \pi t}{T}\right)
$$

where $A$ denotes the amplitude of the oscillation and $T$ denotes the period of turbine's rotation. Load control can be achieved by altering the lift on the rotor blades such that the error between the lift and the desired value for the lift is minimal. Therefore, the error at step $k$ is given by

$$
E^{k}=L^{k}-L_{t a r}
$$

where the desired (target) value for the lift $L_{t a r}$ can be designated by setting $A=0$ in (9) and the lift at step $k$ can be estimated from pressure sensor readings, which can be extracted from the flow model.

\section{B. Load Control}

A combination of two controllers is used to control the lift in this work. The input update is

$$
u_{i}^{k_{c}}=\hat{u}_{i}^{k_{c}}+u^{k}
$$

where: $N_{c}=T / \Delta t$ is the number of steps in one cycle, $k_{c}=0,1, \ldots, N_{c}-1$ is the step within a cycle, $i$ is a trial number, $k=i N_{c}+k_{c}$ is the total number of steps, and $u^{k}$ and $\hat{u}_{i}^{k_{c}}$ are the proportional and phase-lead ILC updates described by (12) and (13) respectively.

$$
\begin{gathered}
u^{k}=\mu_{0} \Delta t E^{k-1} \\
\hat{u}_{i}^{k_{c}}=u_{i-1}^{k_{c}}+\mu_{1} \Delta t E_{i-1}^{k_{c}+\delta}
\end{gathered}
$$

where the error $E$ at step $k$ is calculated from the (10) and $\mu_{0}$ and $\mu_{1}$ are the gains of the proportional and phase-lead ILC controllers respectively.

Remark 1: In this work a constant period and a fixed time step are assumed in the control computation. However, 
variable periods of rotation can be considered by using a constant increment in angle of the blade and varying the time step accordingly. Previous work [21] has shown this produces very similar control behaviour.

The flow contains an oscillatory free stream of equation (9) with $A=0.1, T=2.5$, and the time step $\Delta t=0.005$. Trial and error approach has been used to find the gain value $\mu_{0}$. The best results are obtained for $\mu_{0}=50$. The gain $\mu_{1}$ was initially set to 1 . The performance of such controller is given in Fig. 3(b) (green). It can be observed that the fluctuation in the lift is almost completely damped compared to the uncontrolled case (black).

\section{Actuator dynamics analysis}

The behaviour obtained in the previous subsection is not realistic as it does not include the delay between the controller calculates the required input update and the actuator reaches the required value. To account for this behavior, the response of the actuator is delayed according to

$$
\frac{d \hat{u}}{d t}=\lambda(u-\hat{u})
$$

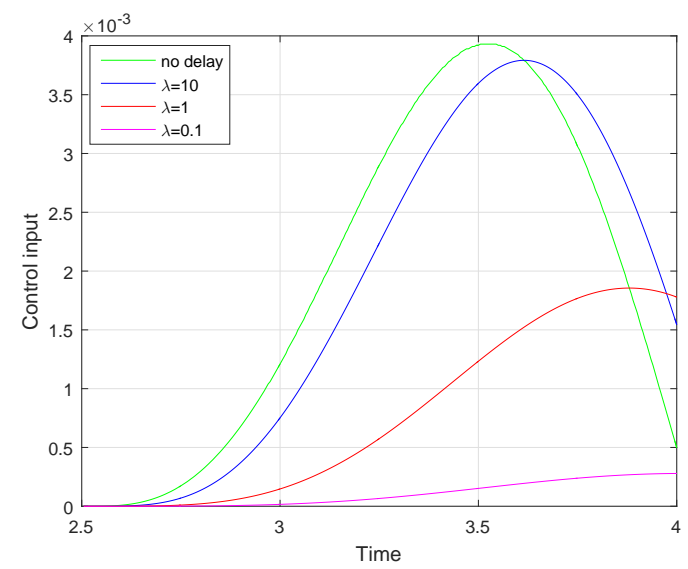

(a) Input signal for different actuator delays $\lambda$

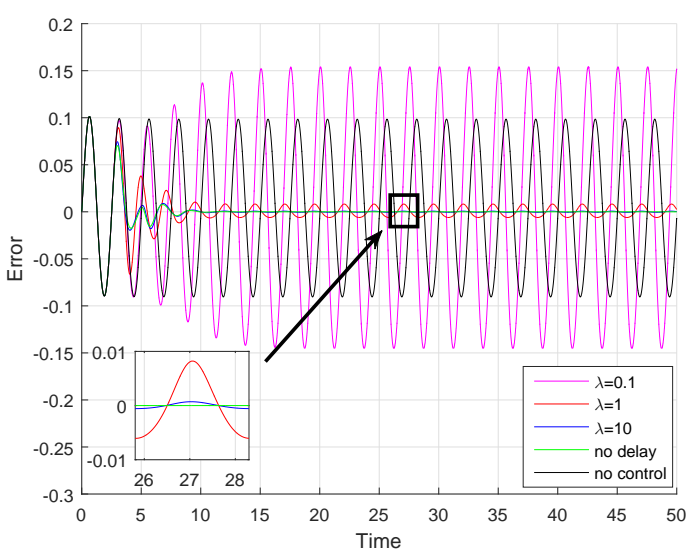

(b) Error signals for different actuator delays $\lambda$

Fig. 3. Input (a) and error (b) signals for different actuator delays where $u$ denotes the input control signal calculated using (11), $\hat{u}$ is the current control signal, and $\lambda$ is the coefficient that determines the speed of response. The control input signal is delayed as can be observed in Fig. 3(a).

The controller with the same gain values as in the previous case $\left(\mu_{0}=50\right.$ and $\left.\mu_{1}=1\right)$ but different speed of response coefficients is now implemented. In this case total damping of the oscillation cannot be achieved. In Fig. 3(b) the error signals obtained for the delayed system with $\lambda=10, \lambda=1$, and $\lambda=0.1$ are given. The error converges to a particular value over early trials, but due to actuation delay, no further decrease is observed. The smaller the value of $\lambda$ the larger the fluctuation in the error, with a very significant fluctuation for $\lambda=0.1$ (magenta). Similar results were obtained in the previous work [15] for a different airfoil type but no solution to the problem was given.

Increasing the value of the gain $\mu_{1}$ is not a solution in this case. In Fig. 4 different values of the gain $\mu_{1}$ are tested for the case when $\lambda=1$. For $\mu_{1}=30$ and $\mu_{1}=40$ significant disturbances can be observed (magenta and red lines in Fig. 4). For $\mu_{1}>40$ the system becomes unstable. In summary, choosing too high gain from the first trial causes significant disturbances and aggravates the performance of the system. Conversely, with lower gain value the oscillatory component remains almost constant after several trials and no further convergence can be observed. The fluctuation which cannot be damped is larger for smaller values of the speed response coefficient $\lambda$.

The main purpose of the remainder of the paper is to examine if trial varying ILC can be used to improve the load performance and damp the lift fluctuations that appear for the model with the delay in actuator response. As demonstrated in this subsection, if the gain of the ILC controller is too high in the first couple trials the disturbances appear. Conversely, if the gain is too small the error converge to a certain value in first several trials but no further convergence is observed. In the next section the controller with the varying gain is implemented in order to examine if the aerodynamic load performance can be improved.

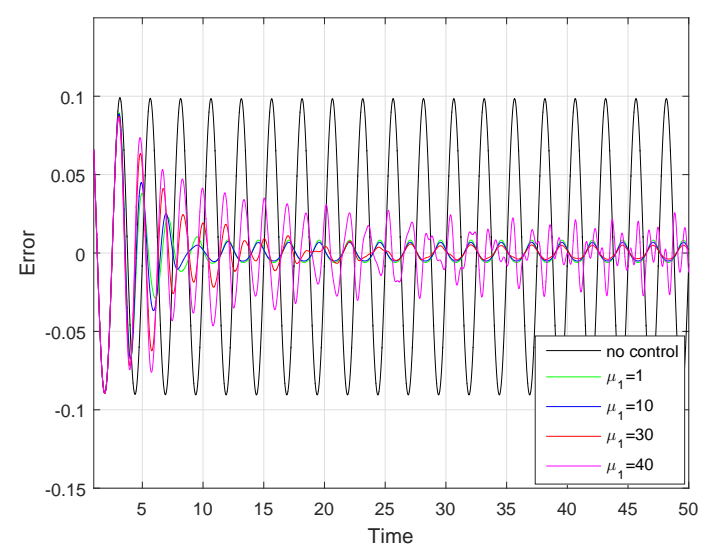

Fig. 4. Error signals for a delay $\lambda=1$ and different $\mu_{1}$ gains 


\section{PERFORMANCE IMPROVEMENT USING TRIAL VARYING ILC}

Based on the results in the previous section, it was decided to examine application of an ILC law with the varying gain that increases with the trial number. The aim of such a controller is to damp the fluctuations in the latter trials without aggravating the performance in the initial trials that can cause the system to become unstable. For the constant gain ILC significant disturbances appear at the beginning of the operation for $\mu_{1} \geq 40$. Conversely, with a smaller gain no disturbances can be observed in the initial trial but the error stops converging after several trials.

The control law now has a form described by equation (11) with the phase-lead ILC component replaced by

$$
\hat{u}_{i}^{k_{c}}=u_{i-1}^{k_{c}}+\mu_{1} \Delta t E_{i-1}^{k_{c}+\delta}
$$

where $\mu_{1}=f(i)$.

A large range of different gain functions have been tested, where in most of them the gain value rises exponentially for approximately $20-30$ trials and then grows logarithmically to prevent it reaching too high a value, with the effect of damaging the system. A selection of the functions are given in Table I and Fig. 5. All tests have been performed for an actuator speed coefficient of $\lambda=1$ and 80 trials to examine if it is possible to achieve further error convergence. Two norms are used to measure the performance. The 2-norm and $\infty$-norm of the error for each trial are calculated using (16).

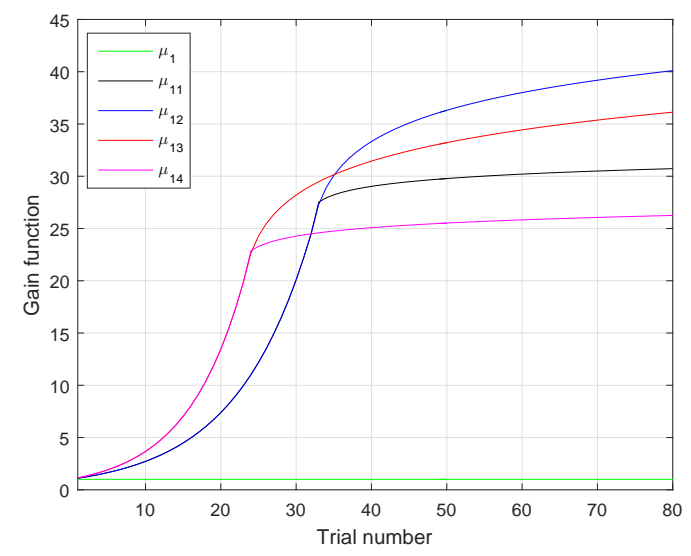

Fig. 5. Different gain functions

TABLE I

GAIN FUNCTIONS

\begin{tabular}{|c|c|}
\hline$\mu_{1}$ & constant $=1$ \\
\hline \multirow[t]{2}{*}{$\mu_{11}$} & $e^{0.1 \cdot i} \quad$ for $i=1: 32$ \\
\hline & $e^{0.1 \cdot 32}+\log (10 \cdot(i-31)) \quad$ for $i=33: 80$ \\
\hline \multirow[t]{2}{*}{$\mu_{12}$} & $e^{0.1 \cdot i} \quad$ for $i=1: 32$ \\
\hline & $e^{0.1 \cdot 32}+4 \cdot \log (i-31) \quad$ for $i=33: 80$ \\
\hline \multirow[t]{2}{*}{$\mu_{13}$} & $e^{0.13 \cdot i} \quad$ for $i=1: 23$ \\
\hline & $e^{0.13 \cdot 23}+\log (10 \cdot(i-22)) \quad$ for $i=24: 80$ \\
\hline \multirow[t]{2}{*}{$\mu_{14}$} & $e^{0.13 \cdot i} \quad$ for $i=1: 23$ \\
\hline & $e^{0.13 \cdot 23}+4 \cdot \log (i-22) \quad$ for $i=24: 80$ \\
\hline
\end{tabular}

The values of the error norms for the last trial and the total 2-norm are given in Table II.

$$
\mathcal{L}_{2}=\sqrt{\frac{1}{N_{c}} \cdot \sum_{k_{c}=1}^{N_{c}}\left(E^{k_{c}}\right)^{2}}, \quad \mathcal{L}_{\infty}=\max \mid E^{k_{c}}
$$

The error norms as a function of the trial number are given

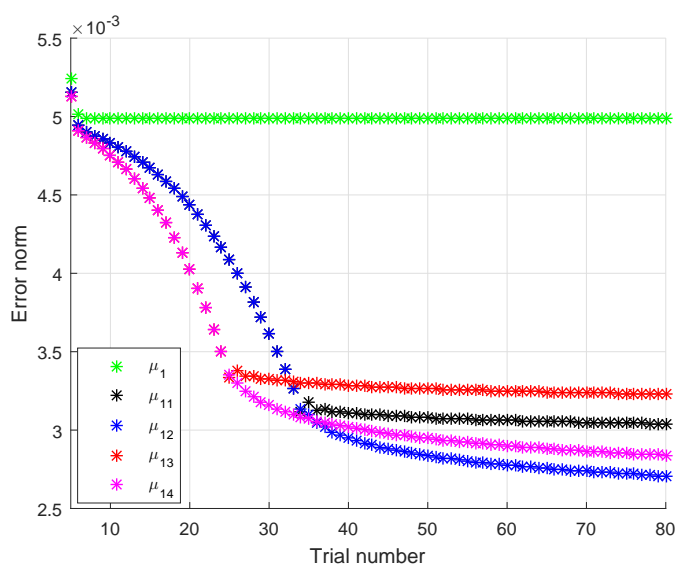

(a) $\mathcal{L}_{2}$ norm for different gain functions

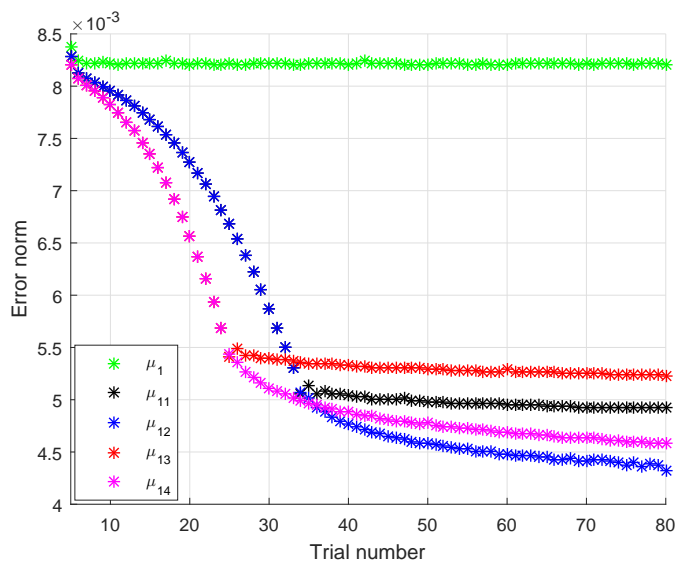

(b) $\mathcal{L}_{\infty}$ norm for different gain functions

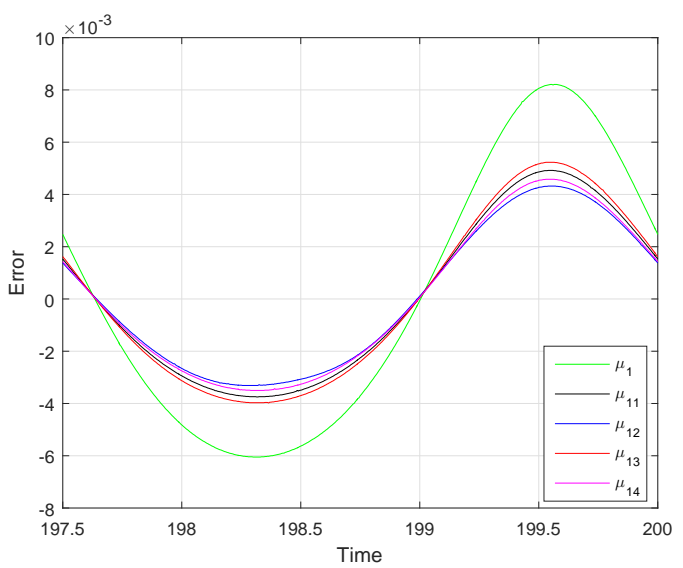

(c) Error signal in the last trial

Fig. 6. The error signal and error norms 
TABLE II

ERROR NORMS AFTER 80 TRIALS

\begin{tabular}{|c|c|c|c|}
\hline gain function & $\mathcal{L}_{2} \cdot 10^{3}$ & $\mathcal{L}_{\infty} \cdot 10^{3}$ & total $\mathcal{L}_{2} \cdot 10^{3}$ \\
\hline$\mu_{1}$ & 5.0 & 8.2 & 379.1 \\
\hline$\mu_{11}$ & 3.0 & 4.9 & 270.8 \\
\hline$\mu_{12}$ & 2.7 & 4.3 & 259.1 \\
\hline$\mu_{13}$ & 3.2 & 5.2 & 271.3 \\
\hline$\mu_{14}$ & 2.8 & 4.6 & 254.6 \\
\hline
\end{tabular}

in the Fig. 6. The results are plotted starting from $i=5$ after the error for the fixed gain ILC stabilizes and no further convergence can be obtained (green). It can be observed that the fluctuations might be decreased with further trials by applying a controller with the trial varying gain $\mu_{11}, \mu_{12}, \mu_{13}$ and $\mu_{14}$. The values of $\mathcal{L}_{2}$ and $\mathcal{L}_{\infty}$ achieved after 80 trials are approximately two times smaller than for the fixed gain ILC (Table II). The total 2-norm for trials $5-80$ decreases by $30-35 \%$ compared to the fixed gain case. The fastest convergence is observed for $\mu_{14}$ and the total 2-norm is the smallest for this case.

\section{CONCLUSIONS AND FUTURE WORK}

In this paper a novel application of trial varying ILC has been investigated for load reduction in wind turbines with smart rotors. A simple CFD model is used to simulate the flow past an airfoil. The flow model includes the oscillatory component of the flow, vortical disturbances and the wake generated from the trailing edge. Circulation generation on the trailing edge is used as a model of smart rotor blade and the combination of proportional controller and the ILC is used to control the loads on the blade. Phase-lead ILC with fixed gain enables a significant reduction in loads to be achieved compared to the uncontrolled case, but when the delay in actuator response is included in the system model the error stops converging after several trials. However, the results in this paper establish that further reduction in the error can be achieved by applying trial varying ILC. Such an approach allows further, monotonic reduction of the fatigue load $\left(\mathcal{L}_{2}\right)$ and peak load $\left(\mathcal{L}_{\infty}\right)$ without aggravating the performance in initial trials or causing the system to become unstable. Increasing the gain slowly as the function of the trial number enables improvement of overall system performance with slower actuator response and both error norms decrease significantly compared to the fixed gain case. However, increasing the gain might aggravate the performance of the system if non-periodic disturbances occur. Model-based design of the of ILC scheme for the wind turbine blade with smart rotor is an obvious step for ongoing research. Such a controller should provide good along the trial performance and trial-to-trial convergence. In this work Kàrmàn-Trefftz airfoil has been used and is the result of a transformation of the circle and modelling via Proper Orthogonal Decomposition (POD) can be performed as the first step to constructing a Reduced Order Model (ROM) of the system [18]. Then, different model-based ILC schemes can be applied with the aim of further improving aerodynamic load performance of wind turbines with smart blades. Tests on various airfoil types, angles of attack and different operating conditions should also be undertaken.

\section{REFERENCES}

[1] L. Y. Pao, K. E. Johnson, "A Tutorial on the Dynamics and Control of Wind Turbines and Wind Farms", Proc. 2009 American Control Conference, St. Louis, MO, USA, 2009, pp. 2076-2089.

[2] S. J. Johnson, C. P. van Dam, D. E. Berg, "Active Load Control Techniques for Wind Turbines", Sandia National Laboratories, Albuquerque, NM, Rep. SAND2008-4809, Aug. 2008.

[3] S. J. Johnson, J. P. Baker, C. P. van Dam, D. E. Berg, ”An Overview of Active Load Control Techniques for Wind Turbines with an Emphasis on Microtabs", Wind Energy, vol. 13, pp. 239-253, Mar. - Apr. 2010.

[4] D. Castaignet, N. K. Poulsen, T. Buhl, Jens J. Wedel-Heinen, "Model Predictive Control of Trailing Edge Flaps on a wind turbine blade", presented at the American Control Conf., San Francisco, CA, USA, 2011.

[5] J. W. Lee, J. H. Han, H. K Shin, H. J. Bang, "Active Load Control of Wind Turbine Blade Section with Trailing Edge Flap: Wind Tunnel Testing", Journal of Intelligent Material Systems and Structures, pp. 1-10, Aug. 2014.

[6] K. Kanistras, M. J. Rutherford, K. P. Valavanis, "Development of a Circulation Control Wing for UAVs", presented at Aerospace Conf., Big Sky, MT, USA, 2014.

[7] T. K. Barlas, G. A. M. van Kuik, "Review of State of the Art in Smart Rotor Control Research for Wind Turbines", Progress in Aerospace Sciences, vol. 46, pp. 1-27, Jan. 2010.

[8] S. S. Collis, R. D. Joslin, A. Seifert, V. Theofilis, "Issues in Active Flow Control: Theory, Control, Simulation, and Experiment", Progress in Aerospace Sciences, vol. 40, pp. 237-289, May 2004.

[9] D. A. Bristow, M. Tharayil, A. G. Alleyne, "A Survey of Iterative Learning Control", IEEE Control Systems Magazine, vol. 26, pp. 96114, Jul. 2006.

[10] H. S. Ahn, Y. Q. Chen, K. L. Moore, ’Iterative Learning Control: Brief Survey and Categorization", IEEE Transactions on Systems, Man, and Cybernetics, Part C (Applications and Reviews), vol. 37, pp. 10991121, Dec. 2007.

[11] L. Hladowski, K. Galkowski, Z. Cai, E. Rogers, C. T, Freeman, P. L. Lewin, "Experimentally supported 2D systems based iterative learning control law design for error convergence and performance", Control Engineering Practice, Vol. 18, 3339-348, 2010.

[12] I. Houtzager, J. van Wingerden, .M. Verhaegen, Rejection of Periodic Wind Disturbances on a Smart Rotor Test Section Using Lifted Repetitive Control, IEEE Trans. Control Syst. Technol., vol. 21, pp. 347-359, Mar. 2013.

[13] O. R. Tutty, M. Blackwell, E. Rogers, R. Sandberg, "Computational Fluid Dynamics based Iterative Learning Control of Peak Loads in Wind Turbines", presented at 51st IEEE Conference on Decision and Control, Maui, HI, USA, 2012.

[14] O. R. Tutty, M. Blackwell, E. Rogers, R. Sandberg, ’Iterative Learning Control for Improved Aerodynamic Load Performance of Wind Turbines With Smart Rotors", IEEE Trans. Control Syst. Technol., vol. 22, pp. 967-979, May. 2014.

[15] M. Blackwell, O. R. Tutty, E. Rogers, R. Sandberg, "Iterative Learning Control Applied to a Non-Linear Vortex Panel Model for Improved Aerodynamic Load Performance of Wind Turbines with Smart Rotors", International Journal of Control, vol. 89, pp. 55-68, 2016.

[16] J. D. Anderson, "Fundamentals of Aerodynamics, Fifth Edition in SI Units", New York, NY:McGraw-Hill, 2011, pp. 282-288.

[17] R. S. Burington, "On the Use of Conformal Mapping in Shaping Wing Profiles", The American Mathematical Monthly, vol. 47, pp. 362-373, Jun. - Jul. 1940.

[18] M. Fahl, 'Trust-region Methods for Flow Control Based on Reduced Order Modelling", Ph.D. dissertation, Trier Univ., Trier, 2000.

[19] N. R. Clarke, O. R. Tutty, "Construction and Validation of a Discrete Vortex Method for the Two-dimensional Incompressible Navier-Stokes Equations", Computer Fluids, vol. 23, pp. 751-783, Jul. 1994.

[20] M. Drela. (1985). Xfoil: An Analysis and Design System for Low Reynolds Number Airfoils. Available at: http://web.mit.edu/drela/Public/papers/xfoil_sv.pdf.

[21] E. Rogers and O. R. Tutty, "Iterative Learning Control with Applications in Energy Generation, Lasers and Healthcare", Proceedings of the Royal Society A, 2016 (in Press). 